\title{
Primary care and the public's health: evidence from service development initiatives
}

The development of primary health care (PHC) has been a central concern of this Journal since it was launched in 2000: 'Implementation of research and evaluation into PHC practice is also an area of significance to the journal and studies that directly address the challenges and successes of implementation are welcomed by the editors. In all papers, authors should demonstrate how their research or development study relates to primary care both in the context of their own country and internationally' (https://www.cambridge.org/core/journals/primaryhealth-care-research-and-development).

In recognition of this concern, the papers in this issue of the journal focus on development and, in this editorial, an overview of the contribution of these papers to the development of PHC will be discussed. The papers included explore two main themes relevant to development: how PHC can best respond to the need to promote health through a recognition of the wider determinants of health and secondly how primary care is best organised to deliver a service which is acceptable and accessible to the population. The papers included in this issue are drawn from studies in Canada, the USA, Nigeria and the UK reflecting the international interest in questions of application and testing of evidence in practice.

The papers by van Weel et al. (2017) and DeSantis et al. (2017) argue for the use of wider frameworks to be used in implementation and evaluation of PHC interventions. van Weel et al. (2017) suggest a focus on the context within which PHC is delivered and identify four dimensions which they advocate should be reported on in studies of PHC: the health system; the social welfare system; the population and society characteristics; and details of the objectives of an intervention. These authors note that most care is delivered in the community and the dimensions identified reflect the reality of the impact of multiple factors, apart from direct care, on people's health. Awareness of these factors suggests the importance of the relationship between PHC, with its access to individuals, and public health, with its (C) Cambridge University Press 2017 focus on the wider determinants (Laverack, 2015). DeSantis et al. (2017) explore a framework drawn from the behavioural sciences in a study testing the application of the Outcomes Rating Scale to adults attending primary care services. The scale asks people to rate themselves in terms of their individual personal wellbeing, their family and close relationships, their social and wider interpersonal relationships, and their overall sense of wellbeing. In this exploratory study, comparing findings with other scales used in primary care to measure depression, they argue that use of this framework has the potential to identify a larger group of people with situational and relationship distress than scales more specifically focussed on disease symptoms. The growing evidence concerning the impact of personal relationships on health supports the use of such a scale in PHC settings (Balfour et al., 2012; Meier, ND).

Underlying this concern with wider factors impacting on health is the world-wide growth in people's experience of living with long-term conditions and multimorbidity. The Cochrane review (Trivedi, 2017) in this issue considers the evidence of the effectiveness of health service and patient orientated interventions on outcomes for people with multimorbidity. Although the evidence suggests benefit from interventions aimed at comorbidity and depression, other findings are mixed and it is concluded that interventions that target problems across conditions are needed, as well as better measurement and research into effectiveness of interventions. Two papers address the intervention and measurement issues, making the case for different, non-medical, types of interventions to address some of the context, behavioural and relationship challenges impacting on health.

Gandy et al. (2017) report on a detailed multimethod evaluation of a multifaceted secondary prevention programme in West Lancashire, UK. This three-year programme consisted of a range of interventions including education sessions, activities such as line dancing, lunch clubs, language 
learning, etc. to promote active ageing amongst people over 50 and reduce further deterioration in their health. The evaluation, which includes quantitative, economic and qualitative data, shows the positive benefits of this multifaceted approach and the benefits achieved particularly through the inter-connections between elements of the interventions. In addition, this paper details the challenges faced in evaluation of these types of initiatives. This issue is also addressed in the paper by Whitelaw et al. (2017) who argue for the need for more evidence concerning the feasibility and benefits of adopting social prescribing into primary care. Social prescribing by primary care of interventions including social activities, arts and crafts, gardening, walking has grown in recent years as reflected in previous papers in this journal (South et al., 2008; Maughan et al., 2016) and reviews relating to specific aspects of social prescribing are increasing (see e.g., Buck, 2016).

The process of implementation of social prescribing or any other change into primary care presents other challenges. The facilitation of the introduction of social prescribing and process evaluation of the implementation in two general practices and a community organisation is the focus of the paper by Whitelaw et al. (2017). These authors demonstrate the value of detailed planning, of understanding the context within which the practices were working, of the role of key change agents and the value of a theoretically driven framework to support implementation and evaluation.

The detailed process of involvement of all staff which contributed to the successful outcomes described by Whitelaw et al. (2017) in the study in Scotland are highlighted further in the study by Brauer et al. (2017) of the development in Ontario of a planning framework for obesity prevention. This process engaged family health teams and patients in the development of a population approach to obesity prevention. This detailed process involving in-depth discussions, ranking exercises, etc. resulted in a shared framework across the whole life span with interventions from awareness raising, to follow-up processes and practice initiatives for different age groups illustrated in figure 2 (Brauer et al., 2017). Returning to the framework proposed by van Weel et al. (2017), Brauer et al. (2017) comment that all the teams involved (part of the health care system) were already interested in the issue of obesity and had delivered initiatives to address this issue. This study highlights the importance of the orientation of PHC team members to the type of service that the PHC team is providing. This second theme, which recognises that the organisation of primary care is critical to issues of acceptability and access, is also addressed in the papers by Ogaji et al. (2017), Wang and Liang (2017), Chang et al. (2017) and Ward (2017).

In a study undertaken by an international team drawn from the Universities of Port Harcourt and Manchester, Ogaji et al. (2017) describe the lack of applicable tools to measure patient evaluations of primary care in sub-Saharan Africa. Drawing on a systematic review and patient interviews they describe in detail the development and validation of a patient evaluation tool and a short form of the tool. These tools, the first of their kind for use in Nigeria, they anticipate will be used in future research and in practice to help evaluate patient-focussed improvements in PHC in Nigeria. These tools therefore add an additional dimension, the patient perspective, to the measurement of the effectiveness of PHC in Nigeria adding to the data, for example, provided at a more organisational level, by the Primary Health Care Performance Initiative for low- and middle-income countries (http://phcperformance initiative.org/about-us/about-phcpi).

Wang and Liang (2017) provide a fascinating, historical account of the rural co-operative medical scheme in China which was built on co-operation between health practitioners and farming communities. Although these schemes largely ended in China the authors point to the growth in cooperative health schemes in less developed and developed economies perhaps suggesting one way in which the prevention and promotion of health really becomes everyone's business. Taking a different approach to participation, Chang et al. (2017) consider participation in health through a study of the acceptability of the use of electronic communication between PHC practitioners and patients. They found that $80 \%$ of the patients surveyed in one Canadian practice had internet access which they used for a wide range of purposes. Staff expressed frustrations with the present communication systems but both groups felt that clear guidelines would need to be put in place to support this form of communication. This description of an environmental scan illustrates an 
additional approach to preparing for and assessing patient and staff reactions to changes which have the potential to improve relations between patients and health practitioners.

Finally, perhaps highlighting an issue regarding communication and co-operation between PHC and a patient group, Ward (2017) discusses data concerning attendance at an emergency department by people with diabetes compared to a control group. Although people with diabetes had more attendances than people in the control group, counter-intuitively and contrary to the $23 \%$ emergency attendance predicted by doctors surveyed, Ward found that only $3.1 \%$ of attendances were for diabetic emergencies. Of the remaining $96 \%$ of attendances many were for problems related to infections: cellulitis, wounds, abscesses. Perhaps reference to the frameworks proposed by van Weel et al. (2017) and DeSantis et al. (2017) might form the basis for future investigation of the reasons for these attendances and provide information to enable primary care to better prevent and treat these non-emergency conditions before people feel the need to attend an emergency department.

The papers included in this issue devoted to development explore two main themes: how PHC can best respond to the need to promote health through a recognition of the wider determinants of health and second, how primary care is best organised to deliver a service which is acceptable and accessible to the population. The papers also illustrate the complexity of introducing change in $\mathrm{PHC}$, for example the impact of different theoretical perspectives, the need to understand patient and staff views before implementation of change and the importance of evaluation to identify elements that might be transferable elsewhere. As identified by Lau et al. (2015) in a systematic review of reviews there is a considerable literature and long history concerning different approaches to implementing change in primary care whether through targeting individual professionals, working at the organisational level or focussing on the context of practice, using one type of intervention or taking a multifaceted approach (Wensing and Grol, 1994; Bryar and Byethway, 1996). The papers in this edition of the journal demonstrate some of these approaches to change and, combined, provide information concerning the process of implementation of change in PHC; methods of evaluation; evidence of effectiveness of interventions, and theory which may contribute to the development of primary care services, support the reorientation of primary care to PHC and public health, and inform future research.

\section{Rosamund Bryar Joint Editor in Chief Emeritus Professor, School of Health Sciences City, University of London Northampton Square, London, UK}

\section{References}

Balfour, A., Morgan, M. and Vincent, C. (editors) 2012. How couple relationships shape our world. London: Karnac Books.

Brauer, P., Royall, D., Dwyer, J., Edwards, A.M., Hussey, T., Kates, N., Smith, H. and Kirkconnell, R. 2017: Obesity services planning framework for interprofessional primary care organizations. Primary Health Care Research and Development 18, 135-47.

Bryar, R. and Bytheway, B. (editors) 1996. Changing primary health care, the teamcare valleys experience. Oxford: Blackwell Science.

Buck, D. 2016. Gardens and health. Implications for policy and practice. London: King's Fud.

Chang, F., Paramsothy, T., Roche, M. and Gupta, N.S. 2017: Patient, staff, and clinician perspectives on implementing electronic communications in an interdisciplinary rural family health practice. Primary Health Care Research and Development 18, 149-60.

DeSantis, B., Jackson, M.J., Duncan, B.L. and Reese, R.J. 2017: Casting a wider net in behavioural health screening in primary care: a preliminary study of the Outcome Rating Scale. Primary Health Care Research and Development 18, 188-93.

Gandy, R., Bell, A., McClelland, B. and Roe, B. 2017: Evaluating the delivery, impact, costs and benefits of an active lives programme for older people living in the community. Primary Health Care Research and Development 18, 122-34.

Lau, R., Stevenson, F., Ong, B.N., Dziedzic, K., Treweek, S., Eldridge, S., Everitt, H., Kennedy, A., Qureshi, N., Rogers, A., Peacock, R. and Murray, E. 2015: Achieving change in primary care-effectiveness of strategies for improving implementation of complex interventions: systematic review of reviews. BMJ Open 5, e009993.

Laverack, G. 2015. Power, empowerment and professional practice, third edition. Basingstoke: Palgrave.

Maughan, D.L., Patel, A., Parveen, T., Braithwaite, I., Cook, J., Lillywhite, R. and Cooke, M. 2016: Primary-care-based social prescribing for mental health: an analysis of financial and environmental sustainability. Primary Health Care Research and Development 17, 114-21. 
Meier, R. no date: What do couple relationships have to do with public health? Policy briefing. Tavistock Relationships, London. Retrieved 8 January 2017 from http://www.tavistockrelation ships.ac.uk/policy-research/policy-briefings/268-relationshipspublic-health-briefing.

Ogaji, D.S., Giles, S., Daker-White, G. and Bower, P. 2017: Development and validation of the patient evaluation scale (PES) for primary health care in Nigeria. Primary Health Care Research and Development 18, 161-82.

South, J., Higgins, T.J., Woodall, J. and White, S.M. 2008: Can social prescribing provide the missing link? Primary Health Care Research and Development 9, 310-18.

Trivedi, D. 2017: Cochrane Review Summary: Interventions for improving outcomes in patients with multimorbidity in primary care and community settings. Primary Health Care Research and Development 18, 109-11.

van Weel, C., Tamblyn, R. and Turnbull, D. 2017: Variation matters and should be included in health care research for comparison of outcomes. Primary Health Care Research and Development 18, 183-87.
Wang, F. and Liang, Y. 2017: China's Rural Cooperative Medical Scheme: a type of health insurance or a type of health cooperative? Primary Health Care Research and Development 18, 194-99.

Ward, M. 2017: Understanding the use of emergency department and urgent care services by diabetic patients of a Family Medicine Health Team: a retrospective observational study. Primary Health Care Research and Development 18, 200-207.

Wensing, M. and Grol, R. 1994: Single and combined strategies for implementing changes in primary care: a literature review. Centre for Reviews and Dissemination, University of York, York. Retrieved 10 January 2017 http://www.crd. york.ac.uk/CRDWeb/ShowRecord.asp?AccessionNumber= 11994008092.

Whitelaw, S., Thirlwall, C., Morrison, C., Osborne, J., Tattum, L. and Walker, S. 2017: Developing and implementing a social prescribing initiative in primary care: insights into the possibility of normalisation and sustainability from a UK case study. Primary Health Care Research and Development $18,112-21$. 